\title{
Analytical Calculation for DC Inductances of Rectangular Spiral Inductors With Finite Metal Thickness in the PEEC Formulation
}

\author{
Jen-Tsai Kuo, Senior Member, IEEE, Ke-Ying Su, Te-Yu Liu, Hao-Hui Chen, Member, IEEE, and \\ Shyh-Jong Chung, Member, IEEE
}

\begin{abstract}
Inductances of rectangular spiral inductors with finite metal thickness are evaluated by the partial element equivalent circuit (PEEC) technique. The six fold partial self and mutual inductance integrals resulting from the PEEC partitions are found to have analytical expressions, so that the singularities of the integrands can be overcome and the theoretical or "exact" results can be obtained very efficiently. Via and underpath required for input/output connection can also be included in the calculation. Calculated inductance values for $\mathbf{2 0}$ square spiral inductors are checked against measured data published in open literature. Dependence of inductance values on metal thickness is investigated for two specific spiral structures.
\end{abstract}

Index Terms-Inductance, partial element equivalent circuit (PEEC), radio frequency integrated circuit (RFIC), spiral inductor.

\section{INTRODUCTION}

I NDUCTORS are essential elements in radio frequency integrated circuits (RFICs) for designing impedance matching networks, oscillators, low noise amplifiers (LNAs), and filters. Due to the structural complexity of a spiral RFIC inductor, accurate computation of its inductance value is usually difficult and time consuming. Thus, many papers are devoted to modeling this circuit element [1]-[8]. Frequency-dependent models for inductors in high ohmic substrate and silicon substrate are developed in [1] and [2], respectively. In [3], silicon IC spiral inductors and transformers are analyzed using electromagnetic analysis. With appropriate approximations, the calculation is reduced to electrostatic and magnetostatic problems. In [4] and [5], closed-form expressions are established by fitting with a large number of data. These expressions are practical but generally not scalable for all parameters. Full-wave electromagnetic methods [6], [7] can offer very accurate solutions; however, they

Manuscript received August 15, 2005; revised October 26, 2005. This work was supported in part by the National Science Council of Taiwan, R.O.C., under Grants NSC 93-2213-E-009-095 and 93-2752-E-009-002-PAE, and in part by the SRC Program under Contract 2003-TJ-1090. The review of this letter was arranged by Associate Editor J.-G. Ma.

J.-T. Kuo, T.-Y. Liu, and S.-J. Chung are with the Department of Communication Engineering, National Chiao Tung University, Hsinchu 300, Taiwan, R.O.C. (e-mail: jtkuo@cc.nctu.edu.tw).

K.-Y. Su was with the Department of Communication Engineering, National Chiao Tung University, Hsinchu 300, Taiwan, R.O.C. He is now with the Taiwan Semiconductor Manufacturing Company (TSMC), Hsinchu 300, Taiwan, R.O.C.

H.-H. Chen is with the Department of Electronic Engineering, Huafan University, Taipei 223, Taiwan, R.O.C.

Digital Object Identifier 10.1109/LMWC.2005.863256 usually take long computation time. In the multiline model [8], based on the theory of multiple coupled lines, numerical complexity in evaluating the inductances of monolithic inductors can be greatly reduced.

At microwave frequencies, the equivalent circuit model of an on-chip inductor is a two-port network consisting of several parasitic capacitors and resistors, in addition to the core inductor [1]-[3]. The major work of modeling an IC inductor is of course to find its inductance. The partial element equivalent circuit (PEEC) technique [9] is suitable to this purpose since it is a circuit-based formulation and numerically equivalent to a full-wave moment method. In the PEEC method, a rectangular spiral inductor is partitioned into pieces of straight conductor bars. For any two partitions, there are self and mutual inductances. The total inductance of the entire inductor is then a sum of all partial self and mutual inductances.

In this letter, analytical expressions for the six-fold integrals, resulting from the PEEC formulation, are used to evaluate inductances of rectangular spiral inductors, which are three-dimensional (3-D) structures since via and underpath are included. Calculated results are checked against the measured data in an existing publication.

\section{Formulation}

The geometric layout of a square spiral inductor shown in Fig. 1 can be specified by number of turns $N$, linewidth $w$, line spacing $s$, metallization thickness $t$ (not shown), and the outer diameter $d$. Assume that the operation frequency is sufficiently low, so that the current density is a constant throughout the conductor, and the evaluation can be performed as a magnetostatic problem. Let the inductor be partitioned into $n$ straight sections, and the $(n-1)$ th and $n$th partitions be via and underpath, respectively, for input/output (I/O) connection. Consider the $k$ th and $m$ th partitions, through the magnetic flux at $\underline{r}_{m}$ due to a current at $\underline{r}_{k}$, the partial mutual inductance $L_{k m}$ can be expressed as a six-fold integral [9]

$$
\begin{aligned}
L_{k m}= & \frac{\mu}{4 \pi} \frac{1}{w_{k} w_{m} t_{k} t_{m}} \\
& \times \int_{t_{k}} \int_{t_{m}} \int_{w_{k}} \int_{w_{m}} \int_{\ell_{k}} \int_{\ell_{m}} \frac{d \underline{\ell}_{k} \cdot d \underline{\ell}_{m}}{\left|\underline{r}_{k}-\underline{r}_{m}\right|} d w_{k} d w_{m} d t_{k} d t_{m}
\end{aligned}
$$

where $\mu$ is the permeability of air. Parameters $w_{k}, t_{k}, \underline{\ell}_{k}$, and $d \underline{\ell}_{k}$ are, respectively, line width, thickness, length vector, and the element vector with the direction along the axis of conductor 


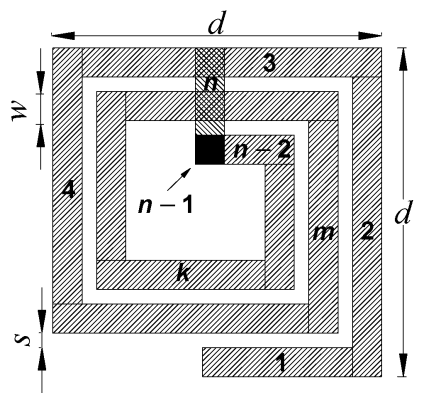

Fig. 1. Partition of a square spiral inductor for PEEC formulation. Via and underpath are also included in the calculation. Finite metallization thickness $t$ is not shown.

segment $k$. When $k=m$, (1) is a partial self inductance $L_{k k}$. The difficulty encountered in evaluating $L_{k k}$ are the singularities resulting from zero denominators. Direct evaluation of (1) may slow down the calculation and deteriorate accuracy of the results. The total inductance of the inductor in Fig. 1 must account for all partial mutual and self inductances, i.e.,

$$
L=\sum_{k=1}^{n} \sum_{m=1}^{n} L_{k m} .
$$

For a rectangular spiral, the term $d \underline{\ell}_{k} \cdot d \underline{\ell}_{m}$ in the numerator of the integrand of (1) is either zero or $\pm d x_{i} d x_{j}$, with $x_{i}$ and $x_{j}$ being $x$ or $y$. The analytical result of the six-fold integral (1) is given as follows [10]:

$$
\begin{aligned}
& \int_{x_{i}}^{x_{i+1}} \int_{x_{j}^{\prime}}^{x_{j+1}^{\prime}} \int_{y_{i}}^{y_{i+1}} \int_{y_{j}^{\prime}}^{y_{j+1}^{\prime}} \int_{z_{i}}^{z_{i+1}} \int_{z_{j}^{\prime}}^{z_{j+1}^{\prime}} \frac{d x d x^{\prime} d y d y^{\prime} d z d z^{\prime}}{\sqrt{\left(x-x^{\prime}\right)^{2}+\left(y-y^{\prime}\right)^{2}+\left(z-z^{\prime}\right)^{2}}} \\
& =\sum_{s=1}^{4} \sum_{t=1}^{4} \sum_{u=1}^{4} c_{s} c_{t} c_{u}\left(f_{1}+f_{2}+f_{3}+f_{4}+f_{5}\right) \\
& f_{1}\left(\alpha_{s}, \beta_{t}, \gamma_{u}\right) \\
& =\frac{\alpha_{s}}{8}\left(\beta_{t}^{2} \gamma_{u}^{2}-\frac{\beta_{t}^{4}+\gamma_{u}^{4}}{6}\right) \times \ln \frac{\Delta+\alpha_{s}}{\Delta-\alpha_{s}} \\
& f_{2}\left(\alpha_{s}, \beta_{t}, \gamma_{u}\right) \\
& =\frac{\beta_{t}}{8}\left(\alpha_{s}^{2} \gamma_{u}^{2}-\frac{\alpha_{s}^{4}+\gamma_{u}^{4}}{6}\right) \times \ln \frac{\Delta+\beta_{t}}{\Delta-\beta_{t}} \\
& f_{3}\left(\alpha_{s}, \beta_{t}, \gamma_{u}\right) \\
& =\frac{\gamma_{u}}{8}\left(\alpha_{s}^{2} \beta_{t}^{2}-\frac{\alpha_{s}^{4}+\beta_{t}^{4}}{6}\right) \times \ln \frac{\Delta+\gamma_{u}}{\Delta-\gamma_{u}} \\
& f_{4}\left(\alpha_{s}, \beta_{t}, \gamma_{u}\right) \\
& =-\frac{\alpha_{s} \beta_{t} \gamma_{u}}{6}\left[f_{a}+f_{b}+f_{c}\right] \\
& f_{a}=\alpha_{s}^{2} \tan ^{-1}\left(\frac{\beta_{t} \gamma_{u}}{\alpha_{s} \Delta}\right) \\
& f_{b}=\beta_{t}^{2} \tan ^{-1}\left(\frac{\alpha_{s} \gamma_{u}}{\beta_{t} \Delta}\right) \\
& f_{c}=\gamma_{u}^{2} \tan ^{-1}\left(\frac{\alpha_{s} \beta_{t}}{\gamma_{u} \Delta}\right) \\
& f_{5}\left(\alpha_{s}, \beta_{t}, \gamma_{u}\right) \\
& =\frac{\Delta^{5}}{60}-\frac{\alpha_{s}^{2} \beta_{t}^{2}+\alpha_{s}^{2} \gamma_{u}^{2}+\beta_{t}^{2} \gamma_{u}^{2}}{12} \Delta
\end{aligned}
$$

TABLE I

Comparison of CALCULATEd INDUCTANCE VALUes With MEASUREd Data

\begin{tabular}{|r|r|r|r|r|r|r|r|r|}
\hline & \multicolumn{1}{|c|}{$\begin{array}{c}d \\
\#\end{array}$} & $\begin{array}{c}w \\
(\mu m)\end{array}$ & $\begin{array}{c}s \\
(\mu \mathrm{m})\end{array}$ & $\begin{array}{c}t \\
(\mu \mathrm{m})\end{array}$ & $\begin{array}{c}L_{\text {mea }} \\
(\mu \mathrm{m})\end{array}$ & $\begin{array}{c}L \\
(\mathrm{nH})\end{array}$ & $\begin{array}{c}\Delta \\
(\%)\end{array}$ \\
\hline 1 & 2.75 & 344 & 29.7 & 1.9 & 0.9 & 3.2 & 2.936 & 8.3 \\
\hline 2 & 3.75 & 292 & 13.0 & 1.9 & 0.9 & 6 & 5.917 & 1.4 \\
\hline 3 & 6.5 & 217 & 5.4 & 1.9 & 0.9 & 12.5 & 11.90 & 4.8 \\
\hline 4 & 2.75 & 279 & 18.3 & 1.9 & 0.9 & 3.1 & 2.964 & 4.4 \\
\hline 5 & 4.75 & 206 & 7.8 & 1.9 & 0.9 & 6.1 & 5.908 & 3.2 \\
\hline 6 & 7.5 & 166 & 3.2 & 1.9 & 0.9 & 12.4 & 11.65 & 6.1 \\
\hline 7 & 9.5 & 153 & 1.8 & 1.9 & 0.9 & 18.2 & 17.35 & 4.7 \\
\hline 8 & 2.75 & 277 & 18.3 & 0.8 & 0.9 & 3.1 & 3.021 & 2.5 \\
\hline 9 & 2.75 & 307 & 18.3 & 18. & 0.9 & 2.9 & 2.413 & 16.8 \\
\hline 10 & 3.75 & 321 & 26.5 & 1.9 & 0.9 & 6.1 & 5.905 & 3.2 \\
\hline 11 & 7.75 & 225 & 4.4 & 1.9 & 0.9 & 18.1 & 17.64 & 2.5 \\
\hline 12 & 3.75 & 193 & 9.1 & 1.9 & 0.9 & 4 & 3.598 & 10.1 \\
\hline 13 & 5 & 171 & 5.4 & 1.9 & 0.9 & 6.1 & 5.677 & 6.9 \\
\hline 14 & 3.25 & 400 & 31.6 & 1.9 & 0.9 & 4.9 & 4.389 & 10.4 \\
\hline 15 & 5.75 & 339 & 10.0 & 1.9 & 0.9 & 16.2 & 15.43 & 4.7 \\
\hline 16 & 12 & 180 & 3.2 & 2.1 & 0.9 & 20.5 & 19.99 & 2.5 \\
\hline 17 & 7 & 300 & 13.0 & 7.0 & 0.9 & 8 & 7.473 & 6.6 \\
\hline 18 & 6 & 400 & 24.0 & 7.0 & 3.0 & 8 & 7.085 & 11.4 \\
\hline 19 & 8 & 300 & 5.0 & 4.0 & 1.0 & 22.1 & 23.38 & -5.8 \\
\hline 20 & 4 & 300 & 5.0 & 4.0 & 1.0 & 9.2 & 9.613 & 8.3 \\
\hline
\end{tabular}

where

$$
\begin{aligned}
& \Delta=\sqrt{\alpha_{s}^{2}+\beta_{t}^{2}+\gamma_{u}^{2}} \\
& \alpha_{1}=x_{i+1}-x_{j+1}^{\prime}, \quad \alpha_{2}=x_{i+1}-x_{j}^{\prime} \\
& \alpha_{3}=x_{i}-x_{j+1}^{\prime}, \quad \alpha_{4}=x_{i}-x_{j}^{\prime} \\
& \beta_{1}=y_{i+1}-y_{j+1}^{\prime}, \quad \beta_{2}=y_{i+1}-y_{j}^{\prime} \\
& \beta_{3}=y_{i}-y_{j+1}^{\prime}, \quad \beta_{4}=y_{i}-y_{j}^{\prime} \\
& \gamma_{1}=z_{i+1}-z_{j+1}^{\prime}, \quad \gamma_{2}=z_{i+1}-z_{j}^{\prime} \\
& \gamma_{3}=z_{i}-z_{j+1}^{\prime}, \quad \gamma_{4}=z_{i}-z_{j}^{\prime} .
\end{aligned}
$$

In (3), $c_{1}=c_{4}=-1$ and $c_{2}=c_{3}=1$. Note that in the derivation of [10] either of the two conductors can be a conducting filament, thin tape, or rectangular bar. The formulas in [11] and [12], for investigating capacitances of charged conducting plates, are special cases of (3)-(15) when both conductors are filaments and thin tapes, respectively. Since the formulation is exact, the theoretical inductance can be calculated very efficiently. Note also that in [10] there is no application of (3)-(15) to calculation of total inductance of a complicated geometry like the spiral inductor shown in Fig. 1.

\section{RESUlTS AND DISCUSSION}

The analytical expressions (3)-(15) are used to calculate inductances for the leading 20 inductors listed in [4] and the results are listed in Table I. In the third through the sixth columns, $d, w, s$, and $t$ are in $\mu \mathrm{m}$. The measured data are list in column of $L_{\text {mea }}$. The last column lists the relative deviations between the calculated results $L$ and $L_{\text {mea }}$, defined as $\Delta=100 \times\left(L_{\text {mea }}-\right.$ $L) / L_{\text {mea }}$. The oxide layer sandwiched between the two metal layers is $8 \mu \mathrm{m}$. In calculation, the contribution of the vertical via to the total inductance is only its self inductance since its current is orthogonal to those of all other straight partitions. Note that inductor 19 has the largest total length that is close to $7.4 \mathrm{~mm}$. 


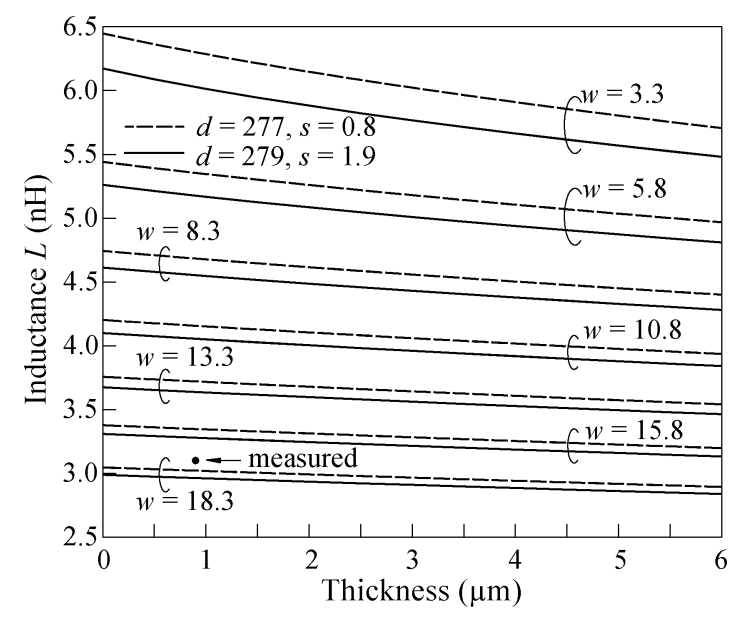

Fig. 2. Dependence of inductance values on the finite metallization thickness. $N=2.75 . d, s$, and $w$ are in $\mu \mathrm{m}$. The black dot shows the measured data of inductors 4 and 8 .

If operation frequency is $300 \mathrm{MHz}$, it is less than $1 \%$ of a wavelength. It is worth mentioning that the CPU time for the 20 inductance values is about $1.3 \mathrm{~s}$ using a $1.8-\mathrm{GHz}$ PC.

As shown in Table I, all calculated values are consistently less than the measured results except inductor 19. There is a trend that inductors with smaller values have larger relative deviations. This could be due to inherent parasitic inductance in the measurement setup [4]. In summary, 10 of the 20 calculated results have $\Delta<5 \%$ and 19 of them have $\Delta<12 \%$, i.e., almost all deviations are within an acceptable extent. Inductor 9 has the largest deviation $\Delta=16.8 \%$. Note that for this inductor the work in [4] also shows a deviation from $10.3 \%$ to $14.6 \%$.

Fig. 2 plots dependence of inductance values of two sets of inductors on their metallization thickness $t$. The two inductors are inductors 4 and 8 in Table I with changes of $w$ and $t$. The results for $t=0$ are obtained by employing the analytical expressions of the four-fold integral in [12]. The inductance value decreases as $t$ or $w$ is increased, as expected. Note that the outer diameters of the two sets of inductors are approximately the same. For these two particular cases, a smaller conductor spacing $s$ results in a larger inductance. As the linewidth is increased, respective effects of varying $s$ and $t$ on the changes of the inductances decrease. The black dot represents the two measured data for inductors 4 and 8 in Table I. Deviations of the two theoretical inductances from the measurements are less than $4.4 \%$.

\section{CONCLUSION}

Inductances of rectangular spiral inductors with finite metallization thickness are evaluated by incorporating analytical expressions for four- and six-fold integrals into the PEEC method. The formulation provides a very simple and efficient way for "exact" dc inductance calculation. The via and underpath required for connection are also taken into account. Results for two specific IC inductor structures show the effectiveness of finite metallization thickness on their inductance values.

\section{REFERENCES}

[1] J. Sieiro, J. M. López-Villegas, J. Cabanillas, J. A. Osorio, and J. Samitier, "A physical frequency-dependent compact model for RF integrated inductors," IEEE Trans. Microw. Theory Tech., vol. 50, no. 1, pp. 384-392, Jan. 2002.

[2] J. Gil and H. Shin, "A simple wide-band on-chip inductor model for silicon-based RF ICs," IEEE Trans. Microw. Theory Tech., vol. 51, no. 9, pp. 2023-2028, Sep. 2003.

[3] A. M. Niknejad and R. G. Meyer, "Analysis, design, and optimization of spiral inductors and transformers for Si RF IC's," IEEE J. Solid-State Circuits, vol. 33, no. 10, pp. 1470-1481, Oct. 2003.

[4] S. S. Mohan, M. d. M. Hershenson, S. P. Boyd, and T. H. Lee, "Simple accurate expressions for planar spiral inductances," IEEE J. Solid-State Circuits, vol. 34, no. 10, pp. 1419-1424, Oct. 1999.

[5] J. R. Long and M. A. Copeland, "Simple accurate expressions for planar spiral inductances," IEEE J. Solid-State Circuits, vol. 32, no. 3, pp. 357-369, Mar. 1997.

[6] F. J. Smückle , "The method of lines for analysis of rectangular spiral inductors," IEEE Trans. Microw. Theory Tech., vol. 41, no. 6/7, pp. 1183-1186, Jun./Jul. 1993.

[7] B. Rejaei, "Mixed-potential volume integral-equation approach for circular spiral inductors," IEEE Trans. Microw. Theory Tech., vol. 52, no. 8, pp. 1820-1829, Aug. 2004.

[8] M. Dehan, J.-P. Raskin, I. Huynen, and D. Vanhoenacker-Janvier, “An improved multiline analysis for monolithic inductors," IEEE Trans. Microw. Theory Tech., vol. 51, no. 1, pp. 100-108, Jan. 2003.

[9] A. E. Ruehli, "Inductance calculations in a complex integrated circuit environment," IBM J. Res. Develop., vol. 16, pp. 470-481, Sep. 1972.

[10] C. Hoer and C. Love, "Exact inductance equations for rectangular conductors with applications to more complicated geometries," J. Res., Nat. Bur. Stand. C., vol. 69C, no. 2, pp. 127-137, Apr.-Jun. 1965.

[11] R. Bancroft, "A note on the moment method solution for the capacitance of a conducting flat plate," IEEE Trans. Antennas Propag., vol. 45, no. 11, p. 1704, Nov. 1997.

[12] J.-T. Kuo and K.-Y. Su, "Analytical evaluation of the MoM matrix elements for the capacitance of a charged plate," IEEE Trans. Microw Theory Tech., vol. 50, no. 5, pp. 1435-1436, May 2002. 